\title{
Prediction of fracture risk and prophylactic intervention in metastatic bone disease: a systematic review
}

\author{
Bogdan Ştefan Crețu* **, Călin Dragosloveanu *, Dragoş Cotor *, \\ Şerban Dragosloveanu ***, Cristian Ioan Stoica* ** \\ * "Foişor" Orthopaedics-Traumatology and Osteoarticular TB Hospital, Bucharest, Romania; \\ ** "Carol Davila" University of Medicine and Pharmacy, Bucharest, Romania
}

Correspondence to: Bogdan Stefan Cretu, MD, PhD, "Foisor" Orthopaedics-Traumatology and Osteoarticular TB Hospital, Bucharest, 35-37 Ferdinand I Blvd., Bucharest, Romania, Mobile Phone: +40741 127 187, E-mail: jfrbogdan@yahoo.com

\begin{abstract}
Pathological fractures occur in an area of bone where either the quantity or quality of bone is modified and the main cause of bone metastases that weaken the structure and will lead to fractures are in high proportion given by visceral tumors or primary hematopoietic tumors like myeloma.

This paper's objective was to review the actual knowledge in the treatment of fractures secondary to metastases. Spinal lesions were not discussed in this paper.

Literature search was performed using MEDLINE and Web of Science to find literature relevant to fracture risk and prophylactic intervention in metastatic bone disease. The Preferred Reporting Items for Systematic Reviews and Meta-Analysis (PRISMA) guideline was used for this review. As results, we identified 30 papers that were suitable for this review. Most of them concluded that it is difficult to assess the amount of bone involvement on radiographs alone. Using the actual guidelines for prophylactic fixation may result in an under treatment or overtreatment of patients with metastatic bone disease. Their ability to determine which metastatic bone lesions will fracture is altered mainly because of the small number of patients included in the studies. The prediction factors for fracture risk are still to be evaluated. CT, FDG-PET or CT scan-based finite element analysis may be useful tools for the identification of impending pathological fractures requiring prophylactic stabilization.

Keywords: bone metastases, pathological fractures, prophylactic stabilization, radiographic evaluation
\end{abstract}

\section{Introduction}

Pathological fractures occur in an area of bone where either the quantity or quality of bone is modified. The main cause of bone metastases that weaken the structure and will lead to fractures are in high proportion given by visceral tumors or primary hematopoietic tumors like myeloma. The outcome of the lesion depends on the bone type and location of the lesion. Patients with bone lesions fall into two categories: previously diagnosed bone tumor that progressed or the first presentation. A primary tumor of the bone should be excluded 
in a patient with no previous diagnosis of malignancy and a current bone lesion. The next step is to stage the tumor, locate the primary lesion and general investigations. For further treatment and decision, the information should be discussed with a multidisciplinary team.

The life expectancy is very difficult to predict in such cases; the management of each patient is highly influenced by the prognosis. The treatment of metastatic disease has multiple goals that need achievement, such as providing pain relief, restore the functionality or preserve it and add a fixation with no need for another surgical intervention. No surgical procedure that provides fixation would be indicated for patients with a short life expectancy, in all other cases the indication should be endoprosthetic replacement due to high failure of internal fixation. The most frequent area treated with endoprosthetics is the proximal femur. Diaphysis lesions are in most cases treated with intramedullary nailing.

When a fracture occurs in an abnormal bone, it is defined as a pathological fracture. The fractures could appear after traumatic events or during normal activities. The main reason for pathological fractures to appear is a metabolic bone disease like osteoporosis, which is the most common, osteomalacia and Paget disease. Congenital diseases, as for example osteogenesis imperfecta, are also included. Besides metabolic bone disease, pathological fractures may take place in bones with benign tumors, like simple bone cyst, malignant primary bone tumors, metastases from visceral primary lesions in the bone structure and hematopoietic primary tumors.

This paper's objective was to review the actual knowledge in the treatment of fractures secondary to metastases. Spinal lesions were not discussed in this paper.

In most patients, a pathological bone fracture through a neoplasm will lead to a metastatic disease. Skeletal metastases may appear many years after the primary tumor was diagnosed. They could be found with the primary lesion at the same time, or they may be the presenting symptoms, which may lead to the discovery of a primary tumor.

The main 5 tumors that lead to bone metastases most frequently are breast, prostate, lung, renal and thyroid. A study made by Harrington, demonstrated postmortem that the majority of breast and prostate tumors also had bone lesions [15]. Patients with high grade prostate or breast cancer will develop metastases in almost $50 \%$ of the cases. Among those patients, 20\% may require orthopedic intervention [29]. For the patients diagnosed with breast cancer, the bone is the most frequent area of distant relapse [3]. Many cases have been identified with skeletal lesions, mainly diagnosed with adenocarcinoma or squamous cell carcinoma, in which the primary tumor is never found but the diagnosis can be established with the use of immunohistochemistry.

The most common sites with metastases are the spine, proximal femur, pelvis, ribs, and proximal humerus. At the femur, 50\% of the cases are with femoral neck fractures, followed by subtrochanteric fractures (30\%) and intertrochanteric fractures (20\%).

The patients with metastatic bone disease are very difficult to manage. Almost $50 \%$ of the patients are not able to live more than 6 months after a pathological bone fracture $[8,13,29,30]$. However, if they follow the treatment, the patients can survive for years and they may not need any further surgery. Identifying patients with low life expectancy who may not need massive skeletal reconstruction and avoiding subjecting the patients to additional surgery (implant related) as much as possible is the main challenge of the orthopedic surgeon.

\section{Materials and methods}

Literature search was performed using MEDLINE and Web of Science to find literature relevant to fracture risk and prophylactic intervention in metastatic bone disease. All 
abstracts found via literature search were screened for relevance to the study topics: (1) bone metastases, (2) fracture risk, and (3) prophylactic fixation. The search was done using specific terms and synonyms.

With the use of the PICOS concept (patient, intervention, comparator, outcome, study type), the next specified criteria arose: (1) bone metastases; (2) the use of prophylactic fixation; (3) no comparator; (4) results of prophylactic fixation; (5) no standard study design; (6) a minimum of 20 patients per study [20]. We used the standard recommendation of two independent readers who performed the selection and subsequent extraction process [20]. The Preferred Reporting Items for Systematic Reviews and Meta-Analysis (PRISMA) guideline was used for this review [20].

\section{Results}

We identified 96 papers from the two databases. After exclusion of 25 papers (duplicates), the number reduced to 71.36 were abandoned because of the title and the abstract. In the end, 35 papers were convenient. Two of them were not obtainable, 3 were inappropriate, 30 of them were used (Fig. 1).

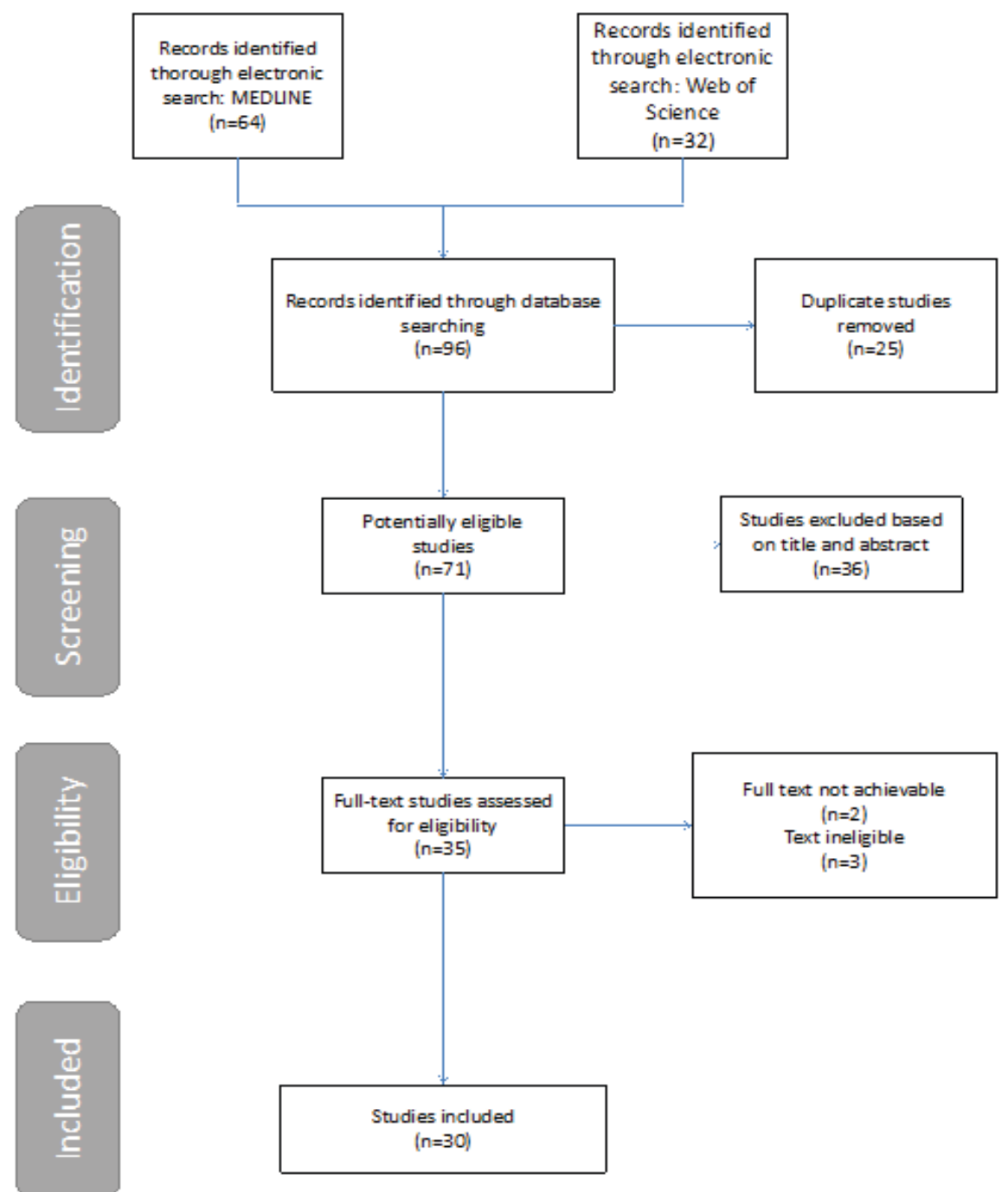

Fig. I Review protocol with the use of PRISMA guideline 
Predicting with high accuracy metastatic bone lesions that can develop a pathological bone fracture would avoid patients' undergoing unnecessary surgery and/ or preventing a fracture. For the estimation of fracture risk, several guidelines and papers have been used that included the size and shape of the bone defect, tumor type, location of the tumor, level of pain and age of patient as variables $[3,5,9,10,18]$. There are different opinions about the most accurate factors that are able to anticipate the risk of fracture. According to Harrington, there should be 4 main factors that may lead to prophylactic bone stabilization to prevent a pathological fracture [15]. These factors included a cortical destruction over $50 \%$, a lesion in the proximal femur $\geq 2.5 \mathrm{~cm}$, a fracture-avulsion in the lesser trochanter, continuous pain despite irradiation. Mirel tried to predict the risk of fracture by using a score that is made of 4 criteria: the character of bone injury, the site, pain and the volume of cortical [19]. A patient with a score of 8 or greater is considered at high risk of fracture that needs prophylactic fixation. In some studies, Mirel score showed a sensitivity of $91 \%$, but a low specificity, 35\% [6].

Unfortunately, Mirel grading has some weak points. His study included only 38 cases that had 78 lesions, fifty of which were secondary to breast cancer. Eleven out of 78 included in the study, were not metastatic lesions. His study promotes prophylactic fixation when a patient has a score $\geq 8$. The cases with a score of 8 had $15 \%$ fracture risk and the cases with a score of 9 had a risk of $33 \%$. For these reasons, a large number of patients could be overtreated with prophylactic fixation.

Current practice shows that pathological fractures are not so common. Multiple studies have reported that only $10 \%$ of the cases had fractures, even with proximal femoral lesions $[1,17,26,29]$.

Assessing the amount of bone involvement is difficult in radiographs. For a noticeable difference, almost $50 \%$ of the cortical bone should be affected. A study that evaluated 203 patients with bone metastatic lesions based on their plain radiographs, found difficulties in assessing the volume of the lesion in $57 \%$, because of the infiltrative character [17].

Another study involving radiographs, undergone on cadavers, found that even without the soft tissue surrounding, the bone consultant orthopedic oncologist had difficulties diagnosing the affected area or determining the size of the bone lesion [14].

On plain radiographs, it is very difficult to measure the defect but even if it was present, we do not know for sure when the fracture will occur based on the destruction.

Some papers showed that the strength reduces almost 60-90\% for a diaphysis defect of $50 \%$ [14]. The decrease in strength for torsional forces had also involved the length rather than the diameter of the defect.

It is outlined that prophylactic fixation has better life expectancy than the treatment of a pathological bone fracture [4,28], due to confounding factors. Pathological bone fractures usually occur in patients with a poor general status and there is a selection error towards prophylactically managed patients, who undergo surgery because they do not have any contraindication for the surgery.

Prophylacticsurgery is considered very often prior to the treatment in skeletal metastases because of the post-radiotherapy fracture risk. In a study, 6 months after radiotherapy, the risk was $35 \%$ [19]. Complications of radiotherapy for long bones are fractures and they increase morbidity and mortality. Prophylactic surgery should be done when the circumferential cortical involvement found on $\mathrm{CT}$ after irradiation is more than $\geq 30 \%$ [23].

Linclau performed osteosynthesis in 36 pathologic fractures, and, in 21 metastases prophylactic internal fixation, the supremacy of the prophylactic rigid osteosynthesis was sustained with the removal of metastatic tissue and the filling up of the bone defect with allograft or methyl methacrylate [18]. Femoral intramedullary nailing of metastatic lesions provides satisfactory results both clinically and radiologically. Early treatment of the metastases prevents fractures and gives 
better results, improving quality of life of these patients [2]. Prophylactic osteosynthesis is also sustained by many other authors $[1,12,16,21,24,27]$. Prophylactic intervention is more cost-effective than the treatment of pathologic fractures in metastatic bone disease [13].

If the conventional X-ray cannot be evaluated, a computed tomography (CT) scan has to be considered. Prophylactic internal fixation of pathological subtrochanteric fractures due to metastatic lesions has to be considered in cases of increasing pain [7]. Fluorodeoxyglucose (FDG)-positron emission tomography (PET) may help identify breast cancer patients at high risk of subsequent pathological fracture of the proximal femur [25]. In the future, CT scan-based finite element (FE) analysis may provide a useful tool for identification of impending pathological fractures requiring prophylactic stabilization $[11,22]$.

\section{Conclusion}

Using the actual guidelines for prophylactic fixation may result in an under treatment or overtreatment of patients with metastatic bone disease. Their ability to determine which metastatic bone lesions will fracture is altered mainly because of the small number of patients included in the studies. The prediction factors for fracture risk are still to be evaluated.

The radiographic evaluation of the bone lesions alone is unable to determine the destructions determined by the metastases and the remaining bone quality. CT, FDG-PET orCT scan-based finite element analysis may be useful tools for the identification of impending pathological fractures requiring prophylactic stabilization.

There is a consensus that prophylactic fixation increases the life expectancy and is more cost-efficient, but this conclusion may be altered by the fact that patients who undergo prophylactic fixation have no contraindications for surgery.

\section{Acknowledgements}

Not applicable.

\section{Conflict of interest statement}

On behalf of all authors, the corresponding author states that there is no conflict of interest.

\section{References}

1. Ampil FL1, Sadasivan KK. Prophylactic and therapeutic fixation of weight-bearing long bones with metastatic cancer. South Med J. 2001 Apr;94(4):394-6.

2. Arvinius C1, Parra JL, Mateo LS, Maroto RG, Borrego AF, Stern LL. Benefits of early intramedullary nailing in femoral metastases. Int Orthop. 2014 Jan;38(1):129-32. doi: 10.1007/s00264-013-2108-x. Epub 2013 Oct 15.

3. Beals RK, Lawton GD, Snell WE. Prophylactic internal fixation of the femur in metastatic breast cancer. Cancer 1971;28:1350-4.

4. Blank AT, Lerman DM, Patel NM, Rapp TB. Is Prophylactic Intervention More Cost-effective Than the Treatment of Pathologic Fractures in Metastatic Bone Disease? Clin Orthop Relat Res. 2016 Jul;474(7):1563-70. doi: 10.1007/s11999-016-4739-x. Epub 2016 Mar 28.

5. Bunting RW. Rehabilitation of cancer patients with skeletal metastases. Clin Orthop Relat Res. 1995 Mar;(312):197-200.

6. Damron TA, Morgan H, Prakash, et al. Critical evaluaton of Mirels rating system of impending pathologic fracture. Clinical Orthopaedics and Related Research 2003;415(Suppl): S201-7.

7. Dijkstra PD, Oudkerk M, Wiggers T. Prediction of pathological subtrochanteric fractures due to metastatic lesions. Arch Orthop Trauma Surg. 1997;116(4):221-4.

8. Dijstra S, Wiggers T, van Geel BN and Boxma H. Impending and actual pathological fractures in patients with bone metastasis of long bones. A retrospective study of 233 surgically treated fractures. European Journal of Surgery 1994;160:535-42.

9. Fidler M: Prophylatic internal fixation of secondary neoplastic deposits in long bones. British Medical Journal 1973;10:341-43.

10. Fidler M. Incidence of fracture of metastasis in long bones. Acta Orthopaedica Scandinavica 1981;52:623-7.

11. Gary A. Ulaner; Alexandra M. Zindman; Junting Zheng; Tae Won B. Kim; John H. Healey. FDG PET/CT Assesses the Risk of Femoral Pathological Fractures in Patients With Metastatic Breast Cancer. Clinical Nuclear Medicine. 42(4):264-270, APR 2017

12. Haentjens P, Casteleyn PP, Opdecam P. Evaluation of impending fractures and indications for prophylactic fixation of metastases in long bones. Review of the liter- 
ature. Acta Orthop Belg. 1993;59 Suppl 1:6-11.

13. Hansen BH, Keller J, Laitinen M, et al. The Scandinavian sarcoma Group skeletal Metastasis Registry. Survival after surgery for bone metastasis in the pelvis and extremities. Acta orthopaedica Scandinavica Supplementum 2004;75:11-15.

14. Hipp JA, Springfield DS, Hayes WC. Predicting pathologic fracture risk in the management of metastatic bone defects. Clinical Orthopaedics and Related Research 1995;312:120-35.

15. Harrington KD. Orthopaedic Management of Metastatic Bone Disease. St Louis, M0: Mosby, 1988.

16. Ibrahim M, Terai H, Yamada K, Suzuki A, Toyoda H, Nakamura $\mathrm{H}$. The role of internal fixation for long bone metastasis prior to impending fracture: an experimental model. J Orthop Sci. 2013 Jul;18(4):659-66. doi: 10.1007/s00776-013-0380-7. Epub 2013 Apr 4.

17. Keene JS, Sellinger DS, McBeath AA, Engber WD. Metastatic breast cancer in the femur. A search for the lesion at risk of fracture. Clinical Orthopaedics and Related Research 1986;203:282.

18. Linclau L, Dokter G. Osteosynthesis of pathologic fractures and prophylactic internal fixation of metastases in long bones. Acta Orthop Belg. 1992;58(3):330-5.

19. Mirel H. Metastatic disease in long bones. Clinical Orthopaedics and Related Research 1989;249:256-64.

20. Moher, D.; Liberati, A.; Tetzlaff, J.; Altman, D.G. PRISM Group. Preferred reporting items for systematic reviews and meta-analyses: The PRISMA statement. BMJ 2009, 339, b2535.

21. Scolaro JA, Lackman RD. Surgical management of metastatic long bone fractures: principles and techniques. J Am Acad Orthop Surg. 2014 Feb;22(2):90-100. doi: 10.5435/JAAOS-22-02-90.

22. Spruijt S1, van der Linden JC, Dijkstra PD, Wiggers T, Oudkerk M, Snijders CJ, van Keulen F, Verhaar JA, Weinans $\mathrm{H}$, Swierstra BA. Prediction of torsional failure in 22 cadaver femora with and without simulated subtrochanteric metastatic defects: a CT scan-based finite element analysis. Acta Orthop. 2006 Jun;77(3):474-81.

23. Tatar Z, Soubrier M, Dillies AF, Verrelle P, Boisgard S, Lapeyre M. Assessment of the risk factors for impending fractures following radiotherapy for long bone metastases using CT scan-based virtual simulation: a retrospective study. Radiat Oncol. 2014 Oct 16;9:227. doi: 10.1186/s13014-014-0227-1.

24. Thompson RC Jr1. Impending fracture associated with bone destruction. Orthopedics. 1992 May;15(5):547-50.

25. Ulaner GA1, Zindman AM, Zheng J, Kim TW, Healey JH. FDG PET/CT Assesses the Risk of Femoral Pathological Fractures in Patients With Metastatic Breast Cancer. Clin Nucl Med. 2017 Apr;42(4):264-270. doi: 10.1097/ RLU.0000000000001580.

26. van der Linden YM1, Kroon HM, Dijkstra SP, Lok JJ, Noordijk EM, Leer JW, Marijnen CA; Dutch Bone Metastasis Study Group. Simple radiographic parameter predicts fracturing in metastatic femoral bone lesions: results from a randomised trial. Radiother Oncol. 2003
Oct;69(1):21-31.

27. Van Geffen E1, Wobbes T, Veth RP, Gelderman WA. Operative management of impending pathological fractures: a critical analysis of therapy. J Surg Oncol. 1997 Mar;64(3):190-4.

28. Wedin R. Surgical treatment for pathologic fracture. Acta Orthop Scand Suppl. 2001 Jun;72(302):2p., 1-29.

29. Wedin R, Bauer HC, Rutqvist LE. Surgical treatment for skeletal breast cancer metastasis: a population-based study of 641 patients. Cancer 2001;92:257-62.

30. Yazawa Y, Frassica F, Chao E, et al. Metastatic bone disease. Clinical Orthopaedics and Related Research 1990;251:213-19. 